\title{
Inapproximability of Survivable Networks *†
}

\author{
Yuval Lando \\ Ben-Gurion University of the Negev \\ E-mail: ylando@hotmail.com
}

\author{
Zeev Nutov \\ The Open University of Israel \\ E-mail: nutov@openu.ac.il
}

December 30, 2008

\begin{abstract}
In the Survivable Network Design Problem (SNDP) one seeks to find a minimum cost subgraph that satisfies prescribed node-connectivity requirements. We give a novel approximation ratio preserving reduction from Directed SNDP to Undirected SNDP. Our reduction extends and widely generalizes as well as significantly simplifies the main results of [9]. Using it, we derive some new hardness of approximation results, as follows. We show that directed and undirected variants of SNDP and of $k$-Connected Subgraph are equivalent w.r.t. approximation, and that a $\rho$-approximation for Undirected Rooted SNDP implies a $\rho$-approximation for Directed Steiner Tree.
\end{abstract}

\section{Introduction}

Let $\kappa_{H}(u, v)$ (possibly $u=v$ ) denote the maximum number of pairwise internally-disjoint $u v$ paths in a directed/undirected graph $H$. Let $\kappa(H)=\min \left\{\kappa_{H}(u, v):(u, v) \in V \times V, u \neq v\right\}$ be the connectivity of $H$. The following is a fundamental problem in Network Design:

Survivable Network Design Problem (SNDP)

Instance: A graph $G=(V, E)$, edge costs $\{c(e): e \in E\}$, and requirements $r(u, v)$ on $V \times V$. Objective: Find a minimum cost spanning subgraph $H=(V, I)$ of $G$ so that

$$
\kappa_{H}(u, v) \geq r(u, v) \quad \text { for all } u, v \in V .
$$

*This research was supported by The Open University of Israel's Research Fund (grant no. 100685).

†Preliminary version in APPROX, LNCS 5171, pages 146-152. 
This formulation includes well known problems such as Steiner Tree/Forest, Min-Cost $k$ Flow, and others. If $r(u, v)=k$ for all $u, v \in V$ then we get the $k$-Connected Subgraph problem, which seeks a minimum cost spanning subgraph $H$ with $\kappa(H) \geq k$. In the Rooted SNDP there is $s \in V$ so that if $r(u, v)>0$ then: $u=s$ for directed graphs, and $u=s$ or $v=s$ for undirected graphs. In $\{0, k\}$-SNDP, requirements are either 0 or $k ;\{0,1\}$-SNDP is the Steiner Forest Problem, and Rooted $\{0,1\}$-SNDP is the Steiner Tree Problem. See a survey in [10] for various types of SNDP problems. The following known statement (c.f., [10]) shows that undirected SNDP problems cannot be much harder to approximate than the directed ones.

Proposition 1.1 A $\rho$-approximation algorithm for Directed SNDP implies a $2 \rho$-approximation algorithm for Undirected SNDP.

The reduction in Proposition 1.1 is very simple: just apply the $\rho$-approximation algorithm on the "bidirected" instance, and return the underlying graph of the directed solution computed.

Following the classification of cost types of [10], we assume that the input graph $G$ to an SNDP instance is complete. The case of 0,1 -costs gives the augmentation problems when we seek to augment a graph $G_{0}$ (formed by edges of cost 0 in $G$ ) by a minimum size edge-set $F$ (any edge is allowed) so that $G_{0}+F$ satisfies the requirements. The case of $1, \infty$-costs gives the min-size subgraph problems: edges in $G$ have cost 1 , while edges not in $G$ have cost $\infty$.

Most undirected variants of $\{0,1\}$-SNDP are substantially easier to approximate than the directed ones. For example, Undirected Steiner Tree/Forest admits a constant ratio approximation algorithm, while the directed variants are not known to admit even a polylogarithmic approximation ratio. Specifically, Dodis and Khanna [4] showed that Directed Steiner Forest is at least as hard to approximate as Label-Cover. By extending the construction of [4], Kortsarz, Krauthgamer, and Lee [9] showed a similar hardness result for Undirected $\{0, k\}$-SNDP; the same hardness is valid even for 0,1 -costs, see [13]. Even a very special case of Directed Steiner Tree - the Group Steiner Tree problem on trees, is unlikely to admit a $\log ^{2-\varepsilon} n$ ratio for any $\varepsilon>0$ [7]. In fact, the best known ratio for Directed Steiner Tree is much worse than its proved lower bound. Extending and simplifying the recursive greedy method introduced by Zelikovsky [16] and Kortsarz and Peleg [12], Charikar et al. [2] gave a combinatorial $O\left(\ell^{3} t^{2 / \ell}\right)$-approximation algorithm for Directed Steiner Tree that runs in $O\left(t^{2 \ell} n^{\ell}\right)$ time, for any integer $\ell \leq n$, where $t$ is the number of terminals to be connected to the root. Substituting $\ell=2 / \varepsilon$ gives an $O\left(t^{\varepsilon}\right)$-approximation scheme, namely, an $O\left(t^{\varepsilon} / \varepsilon^{3}\right)$ approximation algorithm that runs in $O\left(t^{4 / \varepsilon} n^{2 / \varepsilon}\right)$ time for any fixed $\varepsilon>0$. Substituting $\ell=\log t$ gives an $O\left(\log ^{3} t\right)$-approximation in quasi-polynomial time. 
So far, there was no unifying hardness result indicating that the inverse to Proposition 1.1 is also true, namely, that Undirected SNDP is at least as hard to approximate as Directed SNDP. We will give such a reduction, which looks surprisingly simple, after it is found. Our reduction transforms a directed instance on $n$ nodes with costs in the range $C$ into an undirected instance with $n^{\prime}=2 n$ nodes and costs in the range $C^{\prime}=C \cup\{0\}$; hence if the range $C$ includes 0 costs, we have $C=C^{\prime}$. Every requirement $r(u, v)$ transforms into the requirement $r^{\prime}\left(u, v^{\prime}\right)=r(u, v)+n=r(u, v)+n^{\prime} / 2$. We note that the reduction does not preserves metric costs (because we add edges of cost zero), and transforms small requirements into large requirements. However, in several cases, it has the advantage of preserving the "type" of requirements and costs; see [10] for a classification of SNDP problems w.r.t. costs and requirements. In particular, we obtain the following results:

Theorem 1.2 The following holds for any range of costs that includes the zero costs:

(i) A $\rho$-approximation for Undirected SNDP implies a $\rho$-approximation for Directed SNDP.

(ii) A $\rho$-approximation for Undirected $k$-Connected Subgraph implies a $\rho$-approximation for Directed $k$-Connected Subgraph.

(iii) A $\rho$-approximation for Undirected $\{0, k\}$-SNDP implies a $\rho$-approximation for Directed Steiner Forest.

(iv) A $\rho$-approximation for Undirected Rooted $\{0, k\}$-SNDP implies a $\rho$-approximation for Directed Steiner Tree.

We stress again that Theorem 1.2 applies for large values of $k=r_{\max }$, namely, when $k=\Omega(n)$. For small values of $k$ undirected SNDP problems may have better algorithms than the directed ones. This is since $r_{\max }=\Omega(n)$ in the undirected SNDP instance constructed in our reduction. We also note that the theorem applies only for node-connectivity, and not for edge-connectivity; indeed, the edge-connectivity variant of SNDP - so called (undirected) Steiner Network problem admits a 2-approximation algorithm by Jain [8].

To illustrate the power and the limitations of our result, we list and discuss some specific consequences. Note that Theorem 1.2 is true for 0,1 -costs. This fact is however redundant for the problems in parts (ii) and (iii), since for 0,1-costs the directed versions in parts (ii) and (iii) are known to be "easier" than the undirected ones. Specifically, for 0,1-costs we have:

- Directed $k$-Connected Subgraph with 0,1 -costs is in $\mathrm{P}[6]$, while the complexity status of the undirected variant is unknown. 
- Directed Steiner Forest with 0,1-costs admits an $O(\log n)$-approximation [11], while the undirected variant is unlikely to admit a polylogarithmic approximation [13].

Dodis and Khanna [4] showed by a relatively simple proof that Directed Steiner Forest (with general costs) cannot be approximated within $O\left(2^{\log ^{1-\varepsilon} n}\right)$ for any fixed $\varepsilon>0$, unless $\mathrm{NP} \subseteq$ quasi-P. Thus part (iii) immediately implies the main result of [9]:

Corollary $1.3([9])$ Undirected $\{0, k\}$-SNDP does not admit an $O\left(2^{\log ^{1-\varepsilon} n}\right)$-approximation for any fixed $\varepsilon>0$, unless $\mathrm{NP} \subseteq$ quasi-P.

In [13] it is proved that the same hardness result holds even for 0,1-costs, for both directed and undirected graphs (for large values of $k$ ). It seems that this result of [13] cannot be deduced from our work, as the proof of the directed case is essentially the same as that of the undirected one. We also note that the hardness result in Corollary 1.3 was recently improved by Chakraborty, Chuzhoy, and Khanna [1] to a $k^{\varepsilon}$-hardness of approximation for all $k>k_{0}$, where $\varepsilon, k_{0}$ are universal constants. This improved hardness result also cannot be deduced from our reduction.

It was already observed by A. Frank [5] long time ago by an easy proof that Directed Rooted $\{0,1\}$-SNDP with 0,1 -costs is at least as hard as the Set-Cover problem. Hence from part (iv) we obtain the following hardness result from [14].

Corollary 1.4 ([14]) Undirected Rooted $\{0, k\}$-SNDP with 0,1 -costs cannot be approximated within $C \ln n$ for some universal constant $C>0$, unless $\mathrm{P}=\mathrm{NP}$.

Now we give two new results. Combining part (ii) with Proposition 1.1, we obtain:

Corollary 1.5 Directed and undirected variants of SNDP and of $k$-Connected Subgraph are equivalent (up to a constant factor) w.r.t. approximation.

Finally, we can combine part (iv) with the hardness result of Halperin and Krauthgamer [7] for Directed Steiner Tree to obtain:

Corollary 1.6 There exists a constant $C>0$ so that Undirected Rooted $\{0, k\}$-SNDP does not admit a $C \log ^{2-\varepsilon} n$ approximation for any fixed $\varepsilon>0$, unless NP has quasi-polynomial Las-Vegas algorithms.

The hardness in part (iv) however seems "stronger" than the one in Corollary 1.6, as currently no polylogarithmic approximation is known for Directed Steiner Tree. We also note that the current best ratios for Undirected Rooted SNDP are much worse that the ones known for Directed Steiner Tree, see recent papers $[1,3,15]$. 


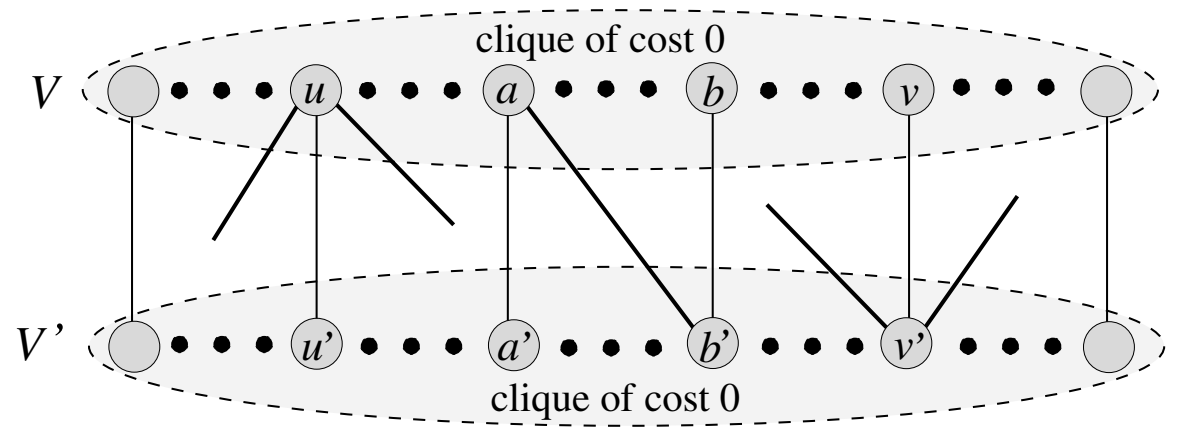

Figure 1: The construction in Lemma 2.1; edges in $M$ are shown by thin lines.

The statements in Corollaries 1.3 - 1.6 are valid even for instances when we are only interested to increase the connectivity by 1 between pairs with requirement $k$, namely, when $G$ contains a subgraph $G_{0}$ of cost 0 with $\kappa_{G_{0}}(u, v)=k-1$ for all $u, v \in V$ with $r(u, v)=k$.

\section{The reduction}

Definition 2.1 Let $H=(V, I)$ be a directed graph. The bipartite (undirected) graph of $H$ is obtained by adding a copy $V^{\prime}$ of $V$ and replacing every directed edge $a b \in I$ by the undirected edge $a b^{\prime}$, where $b^{\prime}$ denotes the copy of $b$ in $V^{\prime}$.

The key observation is the following.

Lemma 2.1 Let $H^{\prime}=\left(V+V^{\prime}, I^{\prime}\right)$ be an undirected graph obtained by adding to the bipartite graph of a directed graph $H=(V, I)$ edge sets of cliques on each of $V$ and $V^{\prime}$, and the matching $M=\left\{z z^{\prime}: z \in V\right\}$ (see Figure 1). Then:

$$
\kappa_{H^{\prime}}\left(u, v^{\prime}\right)=\kappa_{H}(u, v)+n \quad \forall u, v \in V .
$$

Proof: Given a (directed or undirected) graph $J=\left(V_{J}, E_{J}\right)$ and $x, y \in V_{J}$ with $x y \notin E_{J}$, we say that a subpartition $\left(S, S^{*}\right)$ of $V_{J}$ is an xy-separating setpair in $J$ if $x \in S, y \in S^{*}$, and there is no edge from $S$ to $S^{*}$ in $J$ (the set $V_{J}-\left(S+S^{*}\right)$ is an xy-separator). By the node connectivity version of Menger's Theorem

$$
\kappa_{J}(x, y)=\min \left\{\left|V_{J}-\left(S+S^{*}\right)\right|:\left(S, S^{*}\right) \text { is an } x y \text {-separating setpair in } J\right\} .
$$

Now we prove the lemma. We may assume that $u v \notin I$, so $u v^{\prime} \notin I^{\prime}$; otherwise the same proof applies on $H-u v$ and $H^{\prime}-u v^{\prime}$. The following statement establishes a correspondence between $u v^{\prime}$-separating pairs in $H^{\prime}$ and $u v$-separating pairs in $H$. 
Claim: A subpartition $\left(S, S^{\prime}\right)$ of $V+V^{\prime}$ is a $u v^{\prime}$-separating setpair in $H^{\prime}$ if, and only if, $S \subset V, S^{\prime} \subset V^{\prime}$, and $\left(S, S^{*}\right)$ is a $u v$-separating setpair in $H$, where $S^{*}=\left\{z \in V: z^{\prime} \in S^{\prime}\right\}$.

The lemma easily follows from the claim and the version of Menger's Theorem stated above, by observing that $\left|S^{*}\right|=\left|S^{\prime}\right|$ and thus $\left|\left(V+V^{\prime}\right)-\left(S+S^{\prime}\right)\right|=n+\left|V-\left(S+S^{*}\right)\right|$.

It remains to prove the claim. The direction that if $\left(S, S^{*}\right)$ is a $u v$-separating pair in $H$ then $\left(S, S^{\prime}\right)$ is a $u v^{\prime}$-separating pair in $H^{\prime}$ is immediate. We prove the other direction. Assume that $\left(S, S^{\prime}\right)$ is a $u v^{\prime}$-separating pair in $H^{\prime}$, and we prove that then $S \subset V, S^{\prime} \subset V^{\prime}$, and $\left(S, S^{*}\right)$ is a $u v$-separating pair in $H$. Since $v^{\prime} \in S^{\prime}$ and since $v^{\prime}$ is connected to all nodes in $V^{\prime}$, we must have $S \subseteq V$. Similarly, $S^{\prime} \subseteq V^{\prime}$. Clearly, $u \in S$ and $v \in S^{*}$. It remains to prove that $S$ and $S^{*}$ are disjoint; note that since there is no edge between $S$ and $S^{\prime}$ in $H^{\prime}$, this implies that there is no edge from $S$ to $S^{*}$ in $H$, by the construction of $H$. Suppose to the contrary that there is $z \in S \cap S^{*}$. Then $z^{\prime} \in S^{\prime}$. But since $z z^{\prime} \in I^{\prime}$, this contradicts that there is no edge between $S$ and $S^{\prime}$ in $H^{\prime}$.

Corollary $2.2 \kappa\left(H^{\prime}\right)=\kappa(H)+n$ for $H, H^{\prime}$ as in Lemma 2.1.

Proof: Let $k=\kappa(H)$. By Lemma 2.1, it is sufficient to show that:

- $\kappa_{H^{\prime}}(u, v), \kappa_{H^{\prime}}\left(u^{\prime}, v^{\prime}\right) \geq k+n$ for all $u, v \in V$.

- $\kappa_{H^{\prime}}\left(v, v^{\prime}\right) \geq k+n$ for all $v \in V$.

We prove that $\kappa_{H^{\prime}}(u, v) \geq k+n$; the proof that $\kappa_{H^{\prime}}\left(u^{\prime}, v^{\prime}\right) \geq k+n$ is identical. We may again assume that $u v \notin I$. A set of $k+n$ pairwise internally-disjoint $u v$ paths in $H^{\prime}$ is as follows. There are $n-1$ internally-disjoint $u v$-paths in $H^{\prime}-V^{\prime}$. Another path is $u-u^{\prime}-v^{\prime}-v$. We now show additional $k$ paths using nodes from $V^{\prime}-\left\{u^{\prime}, v^{\prime}\right\}$ only. Let $A$ be the set of neighbors of $u$ and $B$ the set of neighbors of $v$ in $V^{\prime}-\left\{u^{\prime}, v^{\prime}\right\}$. We have $|A|,|B| \geq k$, since $H$ is $k$-connected, and since $u v \notin I$. There are $|A \cap B| u v$-paths of the length 2 each through $A \cap B$, and $\min \{|A-B|,|B-A|\}$ uv-paths of the length 3 each through $(A \cup B)-(A \cap B)$. Hence we have $|A \cap B|+\min \{|A-B|,|B-A|\} \geq k$ pairwise internally-disjoint $u v$-paths through $V^{\prime}-\left\{u^{\prime}, v^{\prime}\right\}$, as claimed.

We prove that $\kappa_{H^{\prime}}\left(v, v^{\prime}\right) \geq k+n$. The key point here is that $\kappa_{H}(v, v) \geq k$, namely, that in $H$ there are at least $k$ pairwise internally-disjoint paths from $v$ to itself. Otherwise, by Menger's Theorem, there is a set $C$ with $|C|=k-1$ so that $H-C$ has no path from $v$ to itself. This implies that either $V=C+v$ so $|V|=k$, or that $H-C$ is not strongly connected. In both cases, we obtain the contradiction $\kappa(H) \leq k-1$. Thus by Lemma 2.1 we have $\kappa_{H^{\prime}}\left(v, v^{\prime}\right)=\kappa_{H}(v, v)+n \geq k+n$, as claimed. 
Given an instance $\mathcal{I}=(G=(V, E), c, r)$ of Directed SNDP with $n=|V|$, construct an instance $\mathcal{I}^{\prime}=\left(G^{\prime}=\left(V+V^{\prime}, E^{\prime}\right), c^{\prime}, r^{\prime}\right)$ of Undirected SNDP as follows. $G^{\prime}$ is as in Lemma 2.1, keeping costs of edges in $E$, and with zero costs of other edges in $E^{\prime}$. The requirements are $r^{\prime}\left(u, v^{\prime}\right)=n+r(u, v)$ for $(u, v) \in V \times V$, and $r^{\prime}(u, v)$ or $r^{\prime}\left(u^{\prime}, v^{\prime}\right)$ can be any integer between 0 and $n$ otherwise. Every directed edge in $E$ has a (unique) appearance in $E^{\prime}$, so we identify a directed edge in $E$ with the corresponding undirected edge in $E^{\prime}$. This establishes a bijective correspondence between edge subsets $I \subseteq E$ and edge subsets $I^{\prime} \subseteq E^{\prime}$ containing $E^{\prime}-E$, namely, $I^{\prime}=I+\left(E^{\prime}-E\right)$. From Lemma 2.1 and Corollary 2.2 we get the following statement, which implies Theorem 1.2.

Corollary 2.3 (1) holds for $H=(V, I), r$ if, and only if, (1) holds for $H^{\prime}=\left(V+V^{\prime}, I^{\prime}\right), r^{\prime}$. Furthermore, $\kappa\left(H^{\prime}\right)=\kappa(H)+n$, namely, $H$ is $k$-connected if, and only if, $H^{\prime}$ is $(k+n)$ connected.

Corollary 2.3 implies that $H=(V, I)$ is a feasible solution to instance $\mathcal{I}$ if, and only if, $H^{\prime}=\left(V, I^{\prime}\right)$ is a feasible solution to the constructed instance $\mathcal{I}^{\prime}$; furthermore, $c(I)=c^{\prime}\left(I^{\prime}\right)$, since $I^{\prime}$ is obtained from $I$ by adding edges of cost 0 . In particular, we obtain that the optimal solution values for $\mathcal{I}$ and for $\mathcal{I}^{\prime}$ coincide. This is so both for SNDP and for $k$-Connected Subgraph. Thus if $H^{\prime}=\left(V^{\prime}, I^{\prime}\right)$ is a $\rho$-approximate solution for $\mathcal{I}^{\prime}$, then $H=(V, I)$ is a $\rho$-approximate solution for $\mathcal{I}$. Even if the approximation ratio $\rho$ is given in terms of $n$ (and is non-decreasing in $n)$, then we get an $O(\rho)$-approximation for $\mathcal{I}$, since $\left|V^{\prime}\right|=2|V| \geq|V|$.

As when transforming $\mathcal{I}$ to $\mathcal{I}^{\prime}$, the requirement are shifted by the additive term $n=n^{\prime} / 2$, Directed Steiner Forest is transformed into Undirected $\{0, k\}$-SNDP, while Directed Steiner Tree is transformed into Undirected Rooted $\{0, k\}$-SNDP, where $k=n^{\prime} / 2+1$. Furthermore, an instance of $k$-Connected Subgraph is transformed into an instance of $k^{\prime}$-Connected Subgraph, where $k^{\prime}=n^{\prime} / 2+k$.

Finally, note that since the only change in the range of the costs when transforming $\mathcal{I}$ to $\mathcal{I}^{\prime}$ was adding edges of cost 0 , then the ranges of costs of $\mathcal{I}$ and $\mathcal{I}^{\prime}$ coincide, provided 0 costs are in the range of $\mathcal{I}$.

The proof of Theorem 1.2 is now complete.

Acknowledgment: We thank an anonymous referee for useful comments. 


\section{References}

[1] T. Chakraborty, J. Chuzhoy, and S. Khanna. Network design for vertex connectivity. In $S T O C$, pages $167-176,2008$.

[2] M. Charikar, C. Chekuri, T. Cheung, Z. Dai, A. Goel, S. Guha, and M. Li. Approximation algorithms for directed Steiner problems. Journal of Algorithms, 33:73-91, 1999.

[3] J. Chuzhoy and S. Khanna. Algorithms for single-source vertex-connectivity. In FOCS, 2008. To appear.

[4] Y. Dodis and S. Khanna. Design networks with bounded pairwise distance. In STOC, pages 750-759, 1999.

[5] A. Frank. Augmenting graphs to meet edge-connectivity requirements. SIAM Journal on Discrete Math, 5(1):25-53, 1992.

[6] A. Frank and T. Jordán. Minimal edge-coverings of pairs of sets. J. on Comb. Theory $B, 65: 73-110,1995$.

[7] E. Halperin and R. Krauthgamer. Polylogarithmic inapproximability. In STOC, pages 585-594, 2003.

[8] K. Jain. Factor 2 approximation algorithm for the generalized Steiner network problem. Combinatorica, 21(1):39-60, 2001.

[9] G. Kortsarz, R. Krauthgamer, and J. R. Lee. Hardness of approximation for vertexconnectivity network design problems. SIAM Journal on Computing, 33(3):704-720, 2004.

[10] G. Kortsarz and Z. Nutov. Approximating minimum-cost connectivity problems. In T. F. Gonzalez, editor, Approximation Algorithms and Metaheuristics. Chapman \& Hall/CRC, 2007.

[11] G. Kortsarz and Z. Nutov. Tight approximation algorithm for connectivity augmentation problems. Journal of Computer and System Sciences, 74:662-670, 2008.

[12] G. Kortsarz and D. Peleg. Approximating the weight of shallow Steiner trees. Discrete Applied Math., 93(2-3):265-285, 1999.

[13] Z. Nutov. Approximating connectivity augmentation problems. In SODA, pages 176$185,2005$. 
[14] Z. Nutov. Approximating rooted connectivity augmentation problems. Algorithmica, 44:213-231, 2006.

[15] Z. Nutov. An almost $O(\log k)$-approximation for $k$-connected subgraphs. In $S O D A$, 2009. To appear.

[16] A. Zelikovsky. A series of approximation algorithms for the acyclic directed Steiner tree problem. Algorithmica, 18(1):99-110, 1997. 